\title{
On a Certain Space of Centrally Symmetric Polyhedra Which is a Reproducing Kernel Hilbert Space
}

\author{
Edward Tutaj ${ }^{1,2}$
}

Received: 7 May 2019 / Accepted: 9 September 2019 / Published online: 19 September 2019

(c) The Author(s) 2019

\begin{abstract}
In this paper a certain unitary space will be constructed. The elements of this space can be interpreted as a special type of three dimensional (n-dimensional) polyhedra. Among them one may find, besides the cube, also such elegant mathematical objects as for example the truncated cuboctahedron or the truncated icosidodecahedron. This unitary space, after completion, will turn out to be a reproducing kernel Hilbert space.
\end{abstract}

Keywords Isoperimetric inequality $\cdot$ Hilbert spaces $\cdot$ Reproducing kernels ·

Archimedean polyhedra $\cdot$ Lie groups

Mathematics Subject Classification 46E22

\section{Introduction}

This paper is a continuation of previous papers by the author [8,9], where the old idea of the isoperimetric inequality in the plane was used to construct some reproducing kernel Hilbert space. Namely, let $\Delta=\left[-\frac{\pi}{2}, \frac{\pi}{2}\right]$ and let $K: \Delta \times \Delta \longrightarrow \mathbb{R}$ be a function given by the formula

$$
K(\varphi, \psi)=2-\frac{\pi}{2} \sin |\varphi-\psi| .
$$

As it was proved in [8,9] this function is positive definite and is a reproducing kernel in a Sobolev space $H_{\text {per }}^{1}(\Delta)$. Since $K\left(-\frac{\pi}{2}, \psi\right)=K\left(\frac{\pi}{2}, \psi\right)$, instead of $\Delta$ one may take the unite circle $\mathbb{S}_{1}$ and instead of (1) one may consider an equivalent function defined on $\mathbb{S}_{1} \times \mathbb{S}_{1}$.

$凶 \quad$ Edward Tutaj

edward.tutaj@im.uj.edu.pl

1 Higher Vocational School, Mickiewicza 8, 33-100 Tarnów, Poland

2 Faculty of Mathematics and Computer Science, Jagiellonian University, Łojasiewicza 6, Kraków, Poland 
More exactly, let $<,>$ denote the standard inner product in $\mathbb{R}^{2}$ and let $x, y$ be two unit vectors from $\mathbb{S}_{1}$. Then the function

$$
K_{1}(x, y)=2-\frac{\pi}{2} \sqrt{1-<x, y>^{2}}
$$

is equivalent to (1) in such a sense, that (2) is a reproducing kernel and defines a space isometric to the space defined by (1). It is not hard to see, that one may consider an analogous formula in $\mathbb{R}^{n}$. Indeed, let $\mathbb{S}_{n-1}$ denote the unit sphere in $\mathbb{R}^{n}$, let $<$, > denote the standard inner product in $\mathbb{R}^{n}$ and let $x, y$ be two vectors from $\mathbb{S}_{n-1}$. Consider the function $K_{n-1}: \mathbb{S}_{n-1} \times \mathbb{S}_{n-1} \longrightarrow \mathbb{R}$ given by the formula:

$$
K_{n-1}(x, y)=2-C_{n} \sqrt{1-<x, y>^{2}},
$$

where $C_{n}$ is a suitable, positive constant, which will be discussed later.

One may ask now, if after this extension, the function $K_{n-1}$ remains still positive definite. It turns out, that this is true and the proof of this property for all $n \in \mathbb{N}$ (in particular for $n=3$ ) is the main result of this paper (Theorem 10). The space corresponding to the kernel $K_{n-1}$ is denoted here by $\mathcal{X}^{n}$. It is may be of some interest that the elements of the space $\mathcal{X}^{n}$ can be interpreted as certain centrally symmetric polyhedra like for example the truncated octahedron (in $\mathbb{R}^{3}$ ). As we will see and perhaps this is even more interesting, kernel $K_{n-1}$ "accepts" only the polyhedra with centrally symmetric faces.

The present paper is organized as follows. In the first section we recall some definitions and properties from [8,9] which are necessary to formulate and prove the main result (i.e. Theorem 10). In the next sections we construct a vector space of the so-called Minkowski polyhedra and we define an inner product in this space (formula (23)). The crucial point of this construction is the proof that the quadratic form given (24) is positive definite. In this proof we use the fact, that the special orthogonal group $S O(n)$ is a transitive Lie group. After we define the enlarged kernel function and we check the reproducing property. In the two last sections we present some remarks about the differences between the situation we describe in dimension 2 and the situation in higher dimensions.

\section{Vector Space of Convex Sets}

The information presented in this section is practically the same, as in [8]. However we repeat them here underlying some some differences. In particular in [8] only the case $n=2$ is considered and in this paper we will speak about higher dimensions. Let $<,>$ denote the standard inner product in the Euclidean space $\mathbb{R}^{n}$ and let, as above, $\mathbb{S}_{n-1}$ be the unite sphere in $\mathbb{R}^{n}$. Let $\mathcal{S}$ denote the family of all subsets of $\mathbb{R}^{n}$, which are non-empty, compact and centrally symmetric. In $\mathcal{S}$ we consider the so-called Minkowski addition and the multiplication by positive scalars. We recall below the definitions. Assume that $V, W \in \mathcal{S}, \lambda \geq 0$. We set: 


$$
\begin{aligned}
V+W & =\{v+w: v \in V, w \in W\}, \\
\lambda \cdot V & =\{\lambda \cdot v: v \in V\} .
\end{aligned}
$$

It is not hard to check, that $(\mathcal{S},+, \cdot)$, i.e. the set $\mathcal{S}$ equipped with the operations defined above, is a vector cone.

Clearly, each set $V \in \mathcal{S}$ contains the origin. If such a set $V$ has a non-empty interior, then there is in $\mathbb{R}^{n}$ a norm, say $\|\cdot\|_{V}$, such that $V$ is the unit ball for this norm. However we allow in $\mathcal{S}$ also the sets with empty interiors, i.e. in particular the sets of the form:

$$
I(v, d)=[-d, d] \cdot v
$$

where $v \in \mathbb{S}_{n-1}$ is a unit vector in $\mathbb{R}^{n}$, and $d \geq 0$ is a scalar. The set of the type $I(v, d)$ will be called a diangle.

It is known, that in the cone $(\mathcal{S},+, \cdot)$ holds the so-called cancellation law [6]

$$
(V \in \mathcal{S}, U \in \mathcal{S}, W \in \mathcal{S}) \Longrightarrow(V+W=U+W \Longrightarrow V=W)
$$

A consequence of the properties formulated above is the following theorem, called the Radström embedding theorem [6]. This theorem is quoted in [8] and we quote it here for to introduce some notations.

Proposition 1 There is a unique-up to an isomorphism-vector space $X_{\mathcal{S}}$ called "covering the cone" $\mathcal{S}$. This space is a quotient of the product $\mathcal{S} \times \mathcal{S}$ by the equivalence relation $\diamond$, where:

$$
(U, V) \diamond(P, Q) \Longleftrightarrow U+Q=V+P .
$$

In $X_{\mathcal{S}}$ one defines the operations by obvious formulas:

$$
\begin{aligned}
{[U, V]+[P, Q] } & =[U+P, V+Q], \\
\lambda \geq 0 \Longrightarrow \lambda[U, V] & =[\lambda U, \lambda V] \\
(-1)[U, V] & =[V, U] .
\end{aligned}
$$

One can check, that the operations in $X_{\mathcal{S}}$ are well defined (i.e. do not depend on the choice of the representatives) and that the space $X_{\mathcal{S}}$, defined in such a way, is unique up to an isomorphism.

The map

$$
i: \mathcal{S} \ni U \longrightarrow[(U,\{\theta\})] \in X_{\mathcal{S}}
$$

where $\theta$ is the origin, is called the canonical embedding and instead of $[(U,\{\theta\})]$, we will write simply $U$ and we will also write $\mathcal{S}$ instead of $i(\mathcal{S})$.

We see, that in fact we have a sequence of spaces depending on the dimension of $\mathbb{R}^{n}$. If we would like to concentrate our attention on some particular dimension $k \in \mathbb{N}$, we will use the notation $X_{\mathcal{S}}^{k}$.

The construction described in Proposition 1 can be realized not only in $\mathbb{R}^{n}$, but also in the case of general Banach spaces, or even locally convex spaces and without 
the claiming of central symmetry. We recall here only some facts necessary for our purposes. More details are to be found for example in [4].

Let us recall, that with each element $W \in \mathcal{S}$ one may associate a certain real-valued function defined on $\mathbb{S}_{n-1}$, namely, for $v \in \mathbb{S}_{n-1}$, we set

$$
\bar{W}(v)=\sup \{<v, w>: w \in W\} .
$$

The number $\bar{W}(v)$ is called the width of the (convex) set $W$ with respect to the linear functional $\langle v, \cdot\rangle$. Moreover it is known that the correspondence $W \rightarrow \bar{W}(v)$ is (for each fixed $v$ ) a linear functional on the cone $\mathcal{S}$.

Finally setting for $[U, V] \in X_{\mathcal{S}}$

$$
\overline{[U, V]}(v)=\bar{U}(v)-\bar{W}(v)
$$

we extend this functional from $\mathcal{S}$ to the linear functional on $X_{\mathcal{S}}$.

The correspondence

$$
X_{\mathcal{S}} \ni[U, V] \rightarrow \overline{[U, V]} \in \mathcal{C}\left(\mathbb{S}_{n-1}\right)
$$

is a monomorphism from $X_{\mathcal{S}}$ to the space of continuous functions on $\mathbb{S}_{n-1}$.

As we see, the elements of $X_{\mathcal{S}}$ have a double nature. They are centrally symmetric convex sets and at the same time, they are the real-valued functions defined on $\mathbb{S}_{n-1}$. In the above definitions we used different notations: $W$ (without overline) for a set and $\bar{W}$ (with overline) for the associated function. In the sequel we will use only the notation $W$ (without overline) for a set and for a function because the context always excludes each possible misunderstanding.

We will use in the sequel the diangles defined above. Let us denote by $\mathcal{D}$ the set of all diangles. Clearly this set is closed with respect to the multiplication by positive reals, but it is not a sub-cone of the cone $\mathcal{S}$ (recall, that we work with the Minkowski addition). However we can consider the sub-cone $\mathcal{W} \subset \mathcal{S}$ generated by all diangles understood as the smaller cone containing $\mathcal{D}$. It is easy to check, that $W \in \mathcal{W}$ if and only if $W$ can be written in the form:

$$
W=\sum_{i=1}^{m} I_{i},
$$

where $I_{i} \in \mathcal{D}$ and $m \in \mathbb{N}$. One can easily check, that for each $W \in \mathcal{W}$ the representation (15) is unique. In other words the set of diangles is linearly independent for finite sums. In the sequel it will be more convenient to use a little different notations. Consider a diangle $I(v, d)$ defined above. If $d=1$ we will write $I_{v}$ instead of $I(v, 1)$. Hence we may represent now the elements $W \in \mathcal{W}$ in the form:

$$
W=\sum_{i=1}^{m} d_{i} \cdot I_{v_{i}}
$$


where $v_{i} \in \mathbb{S}_{n-1}$ and $d_{i}>0$. The elements of the form (16) will be called the ordinary or positive or also the Minkowski polyhedra.

Let us remind here that, as we stated above, the set $I_{v}$ is a function given by the formula

$$
I_{v}(w)=\sqrt{1-<v, w>^{2}}
$$

and the polyhedron $W$ has at $w \in \mathbb{S}_{n-1}$ the value

$$
W(w)=\sum_{i} d_{i} I_{v_{i}}(w)
$$

The cone $\mathcal{W}$ depends clearly on the dimension of the space $\mathbb{R}^{n}$, so we will write, if necessary, $\mathcal{W}^{n}$ instead of $\mathcal{W}$. It is clear that when the number of diangles in the representation (15) is smaller than the dimension of space, then the set $W$ has empty interior. However we will call them also polyhedra. Let us now examine some particular cases. If $n=1$ the cone $\mathcal{W}^{1}$ is the set of all centrally symmetric intervals, i.e. $\mathcal{W}^{1}=$ $\{[-r, r]: r \geq 0\}$, hence, as a cone, it is simply $\mathbb{R}^{+}$. If $n=2$ then $\mathcal{W}^{2}$ is the set of all centrally symmetric polygons in $\mathbb{R}^{2}$. In higher dimensions the situation becomes more complicated. It is clear that in $\mathbb{R}^{3}$ the Minkowski sum of three diangles is a parallelepiped and a finite sum of diangles is a centrally symmetric polyhedron. In particular the truncated octahedron is a Minkowski polyhedron. However it is not true, that each centrally symmetric polyhedron is an element of $\mathcal{W}^{3}$. Indeed, the regular octahedron cannot be written as a Minkowski sum of diangles. This follows from some properties of Minkowski polyhedra in dimension three, which may be described as follows: if $W$ is a Minkowski polyhedron, and $F$ is a (two-dimensional) face of $W$, then $F$ is a polygon which has $2 k$ sides for some $k \in \mathbb{N}$ and $k>1$. Clearly, this is not the case of the regular octahedron. We will come back to this question in the last section.

\section{An Inner Product: I}

Let us fix $n \in \mathbb{N}$. The space $X_{\mathcal{S}}^{n}$ described by Proposition 1 is (for $n>2$ ) too big to be the space corresponding to the kernel $K_{n-1}$. We will consider its subspace $X_{\mathcal{W}}^{n} \subset X_{\mathcal{S}}^{n}$ generated by the cone of Minkowski polyhedra, i.e. the polyhedra of the form (16). In other words, the elements of $X_{\mathcal{W}}^{n}$ can be written in the form

$$
W=\sum_{i=1}^{m} x_{i} \cdot I_{v_{i}},
$$

where $x_{i} \in \mathbb{R}$ (i.e. we admit also $x_{i}<0$.) The sums of the form (19) will be called the generalized polyhedra.

Let us notice that $\mathbb{B}_{n} \in \mathcal{S}$, where $\mathbb{B}_{n}$ is the unit ball in $\mathbb{R}_{n}$. Since the unit ball corresponds to the constant function equal 1 , we will write $\mathbf{1}$ instead $\mathbb{B}_{n}$. We are going now to introduce an inner product in the space $\mathcal{X}^{n}=X_{\mathcal{W}}^{n}+\mathbb{R} \cdot \mathbf{1}$. Let us recall (the details are in $[8,9])$, that in the case $n=2$ we have defined in the space $X_{\mathcal{S}}^{2}$ an inner 
product starting from the isoperimetric inequality. This inner product was denoted by $\langle,\rangle_{i}$. In particular if we have two diangles in $\mathbb{R}^{2}$ given by the angles $\varphi$ and $\psi$, (i.e. by the unit vectors $v=(\cos (\varphi), \sin (\varphi))$ and $w=(\cos (\psi), \sin (\psi)))$, then

$$
\left\langle I_{\varphi}, I_{\psi}\right\rangle_{i}=\frac{4}{\pi^{2}}\left(2-\frac{\pi}{2} \cdot \sin |\varphi-\psi|\right)
$$

Suppose now, that we have two generalized polyhedra in $\mathcal{X}^{n}=X_{\mathcal{W}}^{n}+\mathbb{R} \cdot \mathbf{1}$, namely:

$$
W=x_{0} \cdot \mathbf{1}+\sum_{i=1}^{p} x_{j} I_{v_{j}}
$$

and

$$
V=y_{0} \cdot \mathbf{1}+\sum_{j=1}^{q} y_{i} I_{w_{i}}
$$

where $x_{i}, y_{j} \in \mathbb{R}$ (these coefficients are not necessarily positive).

If $\langle$,$\rangle is a bilinear form on \mathcal{X}^{n}$ then

$$
\begin{aligned}
\langle W, V\rangle= & \left\langle x_{0} \cdot \mathbf{1}+\sum_{j=1}^{p} x_{j} I_{v_{j}}, y_{0} \cdot \mathbf{1}+\sum_{i=1}^{q} y_{i} I_{w_{i}}\right\rangle=x_{0} y_{0}\langle\mathbf{1}, \mathbf{1}\rangle+\sum_{j=1}^{p} y_{0} x_{j}\left\langle I_{v_{j}}, \mathbf{1}\right\rangle \\
& +\sum_{i=1}^{q} x_{0} y_{i}\left\langle I_{w_{i}}, \mathbf{1}\right\rangle+\sum_{i, j}\left\langle I_{v_{j}}, I_{w_{i}}\right\rangle x_{j} y_{i}
\end{aligned}
$$

Now we set $\langle\mathbf{1}, \mathbf{1}\rangle=\left\langle\mathbf{1}, I_{v}\right\rangle=1$ for each unit vector $v \in \mathbb{S}_{n-1}$.

Moreover for each two unit vectors $v, w \in \mathbb{S}_{n-1}$ we set

$$
\left\langle I_{v}, I_{w}\right\rangle=2-\sqrt{1-<v, w>^{2}}
$$

Taking into account the formulas (21) and (22) we obtain the following formula for the inner product in $\mathcal{X}^{n}$ :

$$
\langle W, V\rangle=x_{0} y_{0}+\sum_{i=1}^{p} x_{0} y_{i}+\sum_{j=1}^{q} y_{0} x_{j}+\sum_{i, j}\left(2-\sqrt{1-<v, w>^{2}}\right) x_{j} y_{i} .
$$

The quadratic form induced by (23) has the following shape

$$
\|W\|^{2}=\langle W, W\rangle=\left(x_{0}+\sum_{j=1}^{p} x_{j}\right)^{2}+\left(\sum_{j=1}^{p} x_{j}\right)^{2}-\sum_{i, j} \sqrt{1-<v, w>^{2}} x_{i} x_{j} .
$$


One must check now, that this quadratic form is positive definite. We will do it in a few steps. Suppose first that all coefficients are non-negative (i.e. $x_{i} \geq 0$ for all $i \in \mathbb{N}$ ). We have

$$
\left(\sum_{1} x_{j}\right)^{2}-\sum_{i, j} \sqrt{1-<v, w>^{2}} x_{i} x_{j}=\sum_{i, j}\left(1-\sqrt{1-<v, w>^{2}}\right) x_{i} x_{j} \geq 0
$$

since $x_{i} x_{j} \geq 0$ and $1-\sqrt{1-<v, w\rangle^{2}} \geq 0$. Hence if all $x_{i} \geq 0$, then always $\langle W, W\rangle \geq 0$. Moreover if $x_{i} \geq 0$ and $\langle W, W\rangle=0$ then by (25) we have: $x_{0}+\sum_{1} x_{i}=$ 0 . Thus $x_{i}=0$ for all $i \in \mathbb{N}$. The essential step in the definition of the inner product (23) rely on proving that the inequality (25) is valid not only for non-negative, but for all $x_{i} \in \mathbb{R}$. We will do it in the next section.

\section{An Inner Product: II}

Let us start by a number of observations. Suppose that a generalized polyhedron $W$ has the form

$$
W=\sum_{1}^{p} x_{j} I_{v_{j}}
$$

Let us come back to the formula (24). We will define a number of functions on the space of generalized polyhedra. First we define the so-called deficit term as:

$$
D(W)=\left(\sum_{1} x_{j}\right)^{2}-\sum_{i, j} \sqrt{1-<v_{i}, v_{j}>^{2}} x_{i} x_{j}
$$

As we have remarked above

Proposition 2 If $W$ is a positive polyhedron, then $D(W) \geq 0$.

Moreover we denote:

$$
o(W)=\sum_{i=1}^{p} x_{i}
$$

and

$$
m(W)=\sum_{i, j=1}^{p} \sqrt{1-<v_{i}, v_{j}>^{2}} x_{i} x_{j}
$$

The correspondence $W \rightarrow o(W)$ is a linear functional on the space $X_{\mathcal{W}}^{n}$ and the correspondence $W \rightarrow m(W)$ is a quadratic form on $X_{\mathcal{W}}^{n}$. These functionals (up to some factor) are two of the so called mixed volumes which are studied in convex geometry. 
Hence for each $U \in X_{\mathcal{W}}^{n}, V \in X_{\mathcal{W}}^{n}$ we have:

$$
o(U-V)=o(U)-o(V)
$$

and

$$
m(U-V)=2 m(U)+2 m(V)-m(U+V) .
$$

We will use the following remark.

Proposition 3 If $U$ is an ordinary (positive) polyhedron and $t$ is a linear isometry of $\mathbb{R}^{n}$ then $o(U)=o(t(U))$ and $m(U)=m(t(U))$.

Proof Let $U=\sum_{i=1}^{p} x_{i} I_{v_{i}}$ and $x_{i}>0$. Since the linear maps preserve the Minkowski addition and scalar multiplication, then $t(U)=\sum_{1}^{p} x_{i} t\left(I_{v_{i}}\right)=\sum_{1}^{p} x_{i} I_{t\left(v_{i}\right)}$. This means that when $I_{v}$ is a diangle, then $t\left(I_{v}\right)$ is a diangle too. Thus $t(U)$ is a polyhedron with the same coefficients as $U$. Moreover, since $t$ is an isometry then $<v, w>=<t(v), t(w)>$ and this ends the proof.

The Minkowski polyhedra are the special cases of convex and centrally symmetric polyhedra in $\mathbb{R}^{n}$, then the terms like vertex, edge and face of a polyhedron have the usual meaning. Moreover, for each ordinary polyhedron $U$ we may consider a number $s(U)$ defined as the number of all diangles in the representation (16) of $U$. For a pair $(U, V)$ of ordinary polyhedra we set $s(U, V)=s(U-V)$, i.e. $s(U, V)=s(U)+s(V)$ provided that the sets of diangles representing $U$ and $V$ are disjoint.

As it was remarked above, each generalized polyhedron $W \in X_{\mathcal{W}}^{n}$ is a difference of two ordinary polyhedra. In other words, if $W=\sum_{1}^{p} x_{j} I_{v_{j}}$ than, to have $W=U-V$, we set

$$
U=\sum_{x_{j}>0} x_{j} I_{v_{j}},
$$

and

$$
V=\sum_{x_{j}<0}\left(-x_{j}\right) I_{v_{j}} .
$$

The main Proposition of this section may be formulated as follows:

Proposition 4 For each pair $(U, V)$ of ordinary polyhedra, there exist a pair $\left(U^{\prime}, V^{\prime}\right)$ of ordinary polyhedra such that

(i) $o(U-V)=o\left(U^{\prime}-V^{\prime}\right)$

(ii) $m\left(U^{\prime}-V^{\prime}\right) \geq m(U-V)$

(iii) $s\left(U^{\prime}, V^{\prime}\right)<s(U, V)$ i.e. the total number of diangles representing $\left(U^{\prime}, V^{\prime}\right)$ is smaller than the total number of diangles representing $(U, V)$. 
In the proof of this Proposition we will work with the special orthogonal group $S O(n)$. Let us remind, that $S O(n)$ is a compact Lie group. The elements of $S O(n)$ are linear isometries of $\mathbb{R}^{n}$ preserving orientation. For $t \in S O(n)$ and for an ordinary polyhedron $U$, similarly as in [8], we will write $U^{t}$ instead of $t(U)$. Given a pair $(U, V)$ of ordinary polyhedra, we will consider two real-valued functions defined on $S O(n)$. Namely, for $t \in S O(n)$ we set

$$
\Psi_{U V}(t)=\left(o\left(U-V^{t}\right)\right)^{2}-m\left(U-V^{t}\right)
$$

and

$$
\Phi_{U V}(t)=m\left(U+V^{t}\right)
$$

The functions $t \rightarrow \Psi_{U V}, t \rightarrow \Phi_{U V}(t)$ are both continuous on the compact space $S O(n)$, so for each of them there exist in $S O(n)$ the points where they attain their local minima. We will check the following property of the above functions:

Lemma 5 The function $\Phi_{U V}$ attains a local minimum at $t_{o}$ if and only if the function $\Psi_{U V}$ attains a local minimum at $t_{o}$.

Proof Take into account two elements $t$ and $t_{o}$ from $S O(n)$ and suppose that the inequality $\Psi_{U V}(t) \geq \Psi_{U V}\left(t_{o}\right)$ holds. Then, using formulas (27) and (28) we obtain

$$
\begin{aligned}
& {\left[o(U)-o\left(V^{t}\right)\right]^{2}-\left[2 m(U)+2 m\left(V^{t}\right)-m\left(U+V^{t}\right)\right]} \\
& \quad \geq\left[o(U)-o\left(V^{t_{o}}\right)\right]^{2}-\left[2 m(U)+2 m\left(V^{t_{o}}\right)-m\left(U+V^{t_{o}}\right)\right] .
\end{aligned}
$$

It follows from Proposition 3 that the last inequality is equivalent to the inequality $m\left(U+V^{t}\right) \geq m\left(U+V^{t_{o}}\right)$ i.e. to the inequality $\Phi_{U V}(t) \geq \Phi_{U V}\left(t_{o}\right)$.

Now we will recognize a certain property of the function $t \rightarrow \Phi_{U V}(t)=$ $m\left(U+V^{t}\right)$. Let $U=\sum_{i=1}^{p} x_{i} I_{u_{i}}$ and $V=\sum_{j=1}^{q} y_{j} I_{v_{j}}$ be two ordinary (positive) polyhedra. Let $M$ be the bilinear form generating the quadratic form $m$. Hence we have:

$$
\begin{aligned}
\Phi_{U V}(t)= & m\left(U+V^{t}\right)=m(U)+m\left(V^{t}\right)+2 M\left(U, V^{t}\right)=m(U)+m(V) \\
& +\sum_{i, j} \sqrt{1-<u_{i}, t\left(v_{j}\right)>^{2}} x_{i} y_{j} .
\end{aligned}
$$

Setting

$$
\phi_{U V}(t)=\sum_{i, j} \sqrt{1-<u_{i}, t\left(v_{j}\right)>^{2}} x_{i} y_{j}
$$

we may write

$$
\Phi_{U V}(t)=m(U)+m(V)+\phi_{U V}(t)
$$

and then we see that the difference $\Phi_{U V}-\phi_{U V}$ is a constant function. This implies that the local minima of the function $\Phi_{U V}$ are the same as the local minima of the function $\phi_{U V}$. 
The existence of the absolute (local) minima of the function $\phi_{U V}$ is guaranteed by the fact that $\phi_{U V}$ is a real-valued continuous function defined on a compact space $S O(n)$. But for further considerations we will use the fact, that $S O(n)$ is a smooth manifold. Let us denote

$$
\varphi_{i j}(t)=\sqrt{1-<u_{i}, t\left(v_{j}\right)>^{2}}
$$

We observe, that the function $\phi_{U V}$ is a linear combination of the functions $\varphi_{i j}$ with positive coefficients. Looking at formula (33) it is clear, that the function $\varphi_{i j}$ is not differentiable at the point $t \in S O(n)$ if and only if $t\left(v_{j}\right)=u_{i}$. It is also clear, that the set

$$
T_{i j}=\left\{t \in S O(n): t\left(v_{j}\right)=u_{i}\right\}
$$

is closed and has empty interior. Then $\varphi_{i j}$ is a $C^{\infty}$ (even analytic) real function defined on an open and dense subset of $S O(n)$. If a real-valued smooth function $h: \Omega \rightarrow \mathbb{R}$ is defined on an open subset $\Omega \subset \mathbb{R}^{k}$ then one says that $h$ is locally concave when for each $t_{o} \in \Omega$ there exists a convex neighbourhood $U \subset \Omega$ such that $h$ restricted to $U$ is concave. If $h$ is smooth then $h$ is locally concave if and only if at each point $t \in \Omega$ its second derivative $d_{t}^{(2)} h$ is negative definite. This remark make it possible to speak on local convexity (concavity) also for smooth functions defined on open subsets of smooth manifolds. Namely

Definition 6 Let $h: \Omega \rightarrow \mathbb{R}$ be a smooth function defined on an open subset $\Omega$ of a smooth manifold. We will say that $h$ is locally concave (convex) if for each point $t \in \Omega$ the second derivative $d_{t}^{(2)} h$ is negative (positive) definite.

In particular we have the following observation

Proposition 7 For each $u \in \mathbb{S}_{n-1}$, for each $v \in \mathbb{S}_{n-1}$ and for each $t \in S O(n)$ the function

$$
\varphi_{u v}(t)=\sqrt{1-<u, t(v)>^{2}}
$$

is locally concave.

Proof Consider the map $\tau: t \rightarrow t(v) \in \mathbb{S}_{n-1}$. This map is clearly differentiable and $d_{t} \tau$ is linear map from $T_{t}(S O(n))$ to $T_{t(v)}\left(\mathbb{S}_{n-1}\right)$ (the suitable tangent spaces). As we have remarked above, the function $\varphi_{u v}(t)=\sqrt{1-<u, t(v)>^{2}}$ is not differentiable only if $u=t(v)$ and thus it is smooth in an open set. Since the composition $<u, d_{t} \tau>$ is a linear functional on the tangent space $T_{t}$, then the function $\left.t \rightarrow<u, t(v)\right\rangle^{2}$ is locally convex. In consequence $1-<u, t(v)>^{2}$ is locally concave. Since the real function $s \rightarrow \sqrt{s}$ is increasing and concave, then $\varphi_{u v}$ is locally concave.

Summarizing the above considerations we have the following

Proposition 8 Suppose that the function $\Phi_{U V}$ attains the absolute (or local) minimum at the point $t_{o} \in S O(n)$. Then there exist $i \in \mathbb{N}$ and $j \in \mathbb{N}$ such that $t_{o}\left(v_{j}\right)=u_{i}$. 
Proof Let us suppose the opposite, i.e. that for each $i, j \in \mathbb{N}$ we have $t_{o}\left(v_{j}\right) \neq u_{i}$. Hence $t_{o}$ is a point of differentiability of the function $\phi_{U V}$. But the function $\phi_{U V}$ is a linear combination of the functions $\varphi_{i j}$ with positive coefficients. This implies, by Proposition 34, that the function $\phi_{U V}$ is locally concave. In consequence the second derivative of $\Phi_{U V}$ at $t_{o}$ is negative definite. Since the first derivative of $\Phi_{U V}$ at $t_{o}$, by our assumption, is trivial,(i.e. $d_{t_{o}} \Phi \equiv 0$ ), then $\Phi_{U V}$ attains at $t_{o}$ a local maximum. This would imply, that $\Phi_{U V}$ is constant in a neighbourhood of $t_{o}$, which is impossible. This ends the proof of Proposition 8.

Now we are ready to prove Proposition 4.

Proof Let $t_{o}$ be a point such that the function $\Psi_{U V}(t)=\left(o\left(U-V^{t}\right)\right)^{2}-m\left(U-V^{t}\right)$ attains the absolute minimum at $t_{o}$. By Propositions 5 and 8 the polyhedra $U$ and $V^{t_{o}}$ has a common diangle. Thus the difference $U-V^{t_{o}}$ has a smaller number of diangles than $U-V$. This difference $U-V^{t_{o}}$ has a unique representation as a difference $U^{\prime}-V^{\prime}$ of two positive polyhedra without a common diangle. It is not hard to check, that the pair $\left(U^{\prime}, V^{\prime}\right)$ satisfies Proposition 4, and this ends the proof.

We will end this section by proving the theorem announced at the end of the previous section.

Theorem 9 The quadratic form given by formula (24) is positive definite.

Proof Clearly, it is sufficient to show, that the form (26) is positive definite. We know, that this is true, when the polyhedron $W=\sum_{i=1}^{m} x_{i} \cdot I_{v_{i}}$ is positive (i.e. $x_{i} \geq 0$ ). Take now an arbitrary generalized polyhedron $W=\sum_{i=1}^{m} x_{i} \cdot I_{v_{i}}$ where $x_{i}$ are not necessarily positive. Let us represent this polyhedron $W$ as the difference of two positive polyhedra $U$ and $V$, i.e. $W=U-V$. Using Proposition 4 we may find another generalized polyhedron $W^{\prime}=U^{\prime}-V^{\prime}$ represented as the difference of two positive polyhedra $U^{\prime}$ and $V^{\prime}$ in such a way, that $D(W) \geq D\left(W^{\prime}\right)$ and $s\left(W^{\prime}\right)<s(W)$. We may continue this reduction of the number of diangles $s(W)$ to the moment, when the last of polyhedron $W^{\prime}$ such constructed has all coefficients of the same sign. In other words for each generalized polyhedron $W \in X_{\mathcal{W}}$ we can find a positive polyhedron $W^{*}$ such that $D(W) \geq D\left(W^{*}\right)$. But $D\left(W^{*}\right) \geq 0$ since $W^{*}$ is an ordinary polyhedron and this ends the proof of Theorem 9.

\section{The Reproducing Property}

The space we consider, i.e. $\mathcal{X}^{n}$ equipped with the inner product (23) is a unitary space, which - after completion - becomes a Hilbert space. We will denote this completion using the same symbol, namely $\mathcal{X}^{n}$. Analogously as in the case of $\mathcal{X}^{2}$, we will prove, that the spaces $\mathcal{X}^{n}$ are reproducing kernel Hilbert spaces. The necessary information concerning the reproducing kernel Hilbert spaces (RKHS) are to be found in [7].

First, let us recall, that $\mathcal{X}^{n}$ is a function space. This was described above by formulas (13) and (14) and is valid in the whole space $X_{\mathcal{S}}$. Let us recall also, that the diangle 
$I_{v}$ is a function given by the formula $I_{v}(w)=\sqrt{1-<v, w>^{2}}$ and therefore each generalized polyhedron $W=\sum_{i=1}^{m} x_{i} \cdot I_{v_{i}}$ is a function given by the formula

$$
W(w)=\sum_{i=1}^{m} x_{i} I_{v_{i}}(w)=\sum_{i=1}^{m} x_{i} \sqrt{1-<v, w>^{2}} .
$$

Theorem 10 The space $\mathcal{X}^{n}$ with the inner product (23) is a reproducing kernel Hilbert space. The corresponding kernel is the function

$$
K: \mathbb{S}_{n-1} \times \mathbb{S}_{n-1} \ni(x, y) \longrightarrow K(x, y)=2-\sqrt{1-<x, y>^{2}}
$$

Proof For $v \in \mathbb{S}_{n-1}$ the kernel function $k_{v}$ is given by the formula

$$
k_{v}: \mathbb{S}_{n-1} \ni w \longrightarrow 2-\sqrt{1-<v, w>^{2}}
$$

Take into account a function $f(w)=x_{0}+\sum_{1}^{k} x_{j} I_{v_{j}}(w)$. We have

$$
\begin{aligned}
\left\langle k_{v}, f\right\rangle= & \left\langle 2 \cdot \mathbf{1}-I_{v}, x_{0} \cdot \mathbf{1}+\sum_{j=1}^{k} x_{j} I_{v_{j}}\right\rangle=2 x_{0}\langle\mathbf{1}, \mathbf{1}\rangle \\
& +2 \sum_{j=1}^{k} x_{j}\left\langle\mathbf{1}, I_{v_{j}}\right\rangle-x_{o}\left\langle I_{v}, \mathbf{1}\right\rangle-\sum_{j=1}^{k} x_{j}\left\langle I_{v}, I_{v_{j}}\right\rangle .
\end{aligned}
$$

Here, we use the formula $\langle\mathbf{1}, \mathbf{1}\rangle=\left\langle\mathbf{1}, I_{w}\right\rangle=1$ for all $w$ and we obtain that the above equals

$$
\begin{aligned}
x_{o}+\sum_{j=1}^{k} x_{j}-\sum_{j=1}^{k} x_{j} \cdot\left(2-\sqrt{1-<v, v_{j}>^{2}}\right) & =x_{0}+\sum_{j=1} x_{j}-\sum_{j=1}^{k} x_{j}+\sum_{j=1}^{k} x_{j} I_{v_{j}}(v) \\
& =x_{0}+\sum_{j=1}^{k} x_{j} I_{v_{j}}(v)=f(v) .
\end{aligned}
$$

It remains to check that the evaluation functionals are bounded. It follows from (37) that $\left\|k_{v}\right\|^{2}=(2+1)^{2}+1^{2}=10$. Hence we have

$$
\left.|f(v)|^{2}=\left|\left\langle k_{v}, f\right\rangle\right|^{2} \leq\left\|k_{v}\right\|^{2} \cdot\|f\|^{2}=10 \sqrt{1-<I_{v}, I_{v}>^{2}}\right)\|f\|^{2}=10\|f\|^{2}
$$

Hence $|E(f)| \leq \sqrt{10}|| f||$. The first equality follows from the reproducing property. The inequality is a consequence of the Schwarz inequality. This ends the proof of the Theorem (10). 


\section{Vector Order in $X_{\mathcal{S}}$}

The space $X_{\mathcal{S}}$ is partially ordered by the cone $\mathcal{S}$ itself, but we will consider here a weaker order introduced by inclusion. More exactly, we set

$$
\left[U_{1}, V_{1}\right] \leq\left[U_{2}, V_{2}\right] \Longleftrightarrow U_{1}+V_{2} \subset U_{2}+V_{1}
$$

It is easy to check — using the Radströem lemma (see [6]) — that this order is a linear order. We will check, that $X_{\mathcal{S}}$ with the above order is a lattice. First we observe, that (here $\theta$ denote the one point set $\{\theta\}$ ):

$$
\sup ([U, \theta],[V, \theta])=[\operatorname{conv}(U \cup V), \theta] .
$$

It is known, that a linear ordered space $(Y, \leq)$ is a lattice, if and only if for each $x \in Y, y \in Y$ there exists $\sup \{x, 0\}$. Hence, in our case, we must prove, that for each $[U, V] \in X_{\mathcal{S}}$ there exists $\sup \{[U, V], \theta\}$. On the other hand, it is known (in general situation) that $\sup \{x, y\}=\sup \{x-y, 0\}+y$. Hence setting $x=[U, \theta], y=[V, \theta]$ we have $x-y=[U, V]$ and finally we obtain:

$$
\sup \{[U, V], \theta\}+[V, \theta]=\sup \{[U, \theta],[V, \theta]\} .
$$

The formula for "sup" in general situation, which results from the above considerations is the following:

$$
\sup \{[U, V],[P, Q]\}=[\operatorname{conv}((U+Q) \cup(V+P))+P, Q]
$$

Since the last "sup" exists, we have proved the following:

Theorem 11 The space $X_{\mathcal{S}}$ equipped with the inclusion order is a lattice (independently on the dimension).

Now we will concentrate on the special case of dimension $n=2$. Take into account the space $X_{\mathcal{W}}^{2}$. This is a subspace of $X_{\mathcal{S}}^{2}$ and it turns out, that:

Theorem 12 The space $X_{\mathcal{W}}^{2}$ is a sublattice of $X_{\mathcal{S}}^{2}$.

This follows from the observation, that the convex hull of two centrally symmetric polygons is a centrally symmetric polygon and from the fact, that in dimension $n=2$ each such polygon is a Minkowski sum of diangles. The last fact has an easy, but worth to notice, consequence.

Theorem 13 The space $X_{\mathcal{W}}^{2}$ is dense in $X_{\mathcal{S}}^{2}$ with respect to the uniform convergence.

Proof The closure of $X_{\mathcal{W}}^{2}$ is a sublattice of the completion of $X_{\mathcal{S}}^{2}$ with respect to the norm of uniform convergence. But this completion is the space of continuous functions on some compact space. Then the result follows from the Kakutani theorem about the density of sublattices in the Banach space of continuous functions (see [5]). 
As we have observed above, in dimension $n \geq 3$, the linear subspace $X_{\mathcal{W}}^{n}$ is not a sublattice of $X_{\mathcal{S}}^{n}$. For example the regular octahedron is the supremum of three diangles, but is not a Minkowski polyhedron. Maybe $X_{\mathcal{W}}^{n}$ is still dense in the norm of uniform convergence, but to check it we cannot use the theorems like the theorem of Kakutani. However we can prove the following:

Theorem 14 For every dimension $n \in \mathbb{N}$ the unit ball $\mathbb{B}_{n}$ belongs to the closure of $X_{\mathcal{W}}^{n}$ with respect to the norm of uniform convergence.

Proof We write down only the sketch of the proof and only in the case when $n=3$. We use in the proof below the notion of Bochner integral for vector-valued functions. The original paper is that of Bochner [1], but, if necessary, the needed information are to be found elsewhere. Let us consider the unit ball $\mathbb{B}_{3}$, and the unit sphere $\mathbb{S}_{2}$ with the Lebesgue measure $\mu_{2}$ and the interval $[0, \pi]$ with the Lebesgue measure $m_{1}$. Let $Q$ denote the cube described on the unit ball with the edges parallel to the axis and with the distinguished diagonal parallel to the vector $(1,1,1)$. For $x \in \mathbb{S}_{2}$ and $\varphi \in[0, \pi]$ we denote by $Q^{x, \varphi}$ the cube described on $\mathbb{B}_{2}$ with the diagonal parallel to $x$ and turned by the angle $\varphi$. Clearly $Q^{x, \varphi}$ belongs to $X_{\mathcal{W}}^{3}$. Let $U$ be the Bochner integral with respect to the measure $\mu_{2} \times m_{1}$ of the function $(x, \varphi) \longrightarrow Q^{x, \varphi}$. Since $\mu_{2}$ is uniformly distributed, then the width of $U$ is constant on $\mathbb{S}_{2}$. Hence $U$ is a ball having a radius $r>0$ and this ends the proof.

\section{Additional Remarks}

We will end this paper by a number of additional remarks ad comments.

Remark 1 The kernels we consider in this paper are the functions defined on $\mathbb{S}_{n-1} \times$ $\mathbb{S}_{n-1}$. Maybe the quotient of $\mathbb{S}_{n-1}$ by the antipodal map fits better to the situation we consider, but this is an unessential detail.

Remark 2 Theorem 10 can be restated in a slightly stronger version. Namely

Theorem 15 For each $n \in \mathbb{N}$ there exists a constant $C_{n} \geq 1$ such that the function $K_{n-1}: \mathbb{S}_{n-1} \times \mathbb{S}_{n-1} \longrightarrow \mathbb{R}$ given by the formula:

$$
K_{n-1}(x, y)=2-C_{n} \sqrt{1-<x, y>^{2}} .
$$

is a reproducing kernel.

We have proved in fact in Theorem 10, that the constant $C_{n}=1$ is "good" for each dimension. However, for example in [8] it is proved, that for $n=2$ one can take $C_{2}=\frac{\pi}{2}$. The inequalities considered in this paper are the (easy) special cases of the Aleksandrov-Fenchel inequality [3] and perhaps the best constants $C_{n}$ are hidden somewhere in the convex geometry. We should notice here, that that Theorem 10 is a multidimensional version of the so-called Sequence Model from [9]. However we were not able to find an alternative satisfactory description of the space $\mathcal{X}^{n}$ as a kind of 
Sobolev type spaces. Let us remark also, that one can repeat the construction presented in this paper also in infinite dimension replacing the spheres $\mathbb{S}_{n}$ by the unit sphere $\mathbb{S}$ in $l_{2}$ and replacing the group $S O(n)$ by the (transitive) group of isometries of $\mathbb{S}$.

Remark 3 The Minkowski polyhedra have many special properties distinguishing them among the family of all convex polyhedra. They are centrally symmetric, and since the sum of any number of diangles is always a centrally symmetric polyhedron, then each face of a centrally symmetric polyhedron is itself a centrally symmetric polyhedron. It is interesting to observe, that the converse is also true. This is a known result (see [2]), true independently on dimension. We will present here a proof (for $n=3$ ), written in the language of diangles. Namely

Theorem 16 A three dimensional polyhedron $W$ is, up to a translation, a Minkowski polyhedron, if and only if each face of $W$ is a centrally symmetric polygon.

Proof Let $W$ be a polyhedron satisfying the assumption of Theorem 16. We will consider the pairs of the form $(k, S)$, where $k$ is an edge and $S$ is a face of $W$ and moreover $k \subset S$. Given two such pairs $\left(k_{1}, S_{1}\right)$ and $\left(k_{2}, S_{2}\right)$ we define an order as follows

$$
\left(k_{1}, S_{1}\right)<\left(k_{2}, S_{2}\right) \Longleftrightarrow k_{1} \neq k_{2} \wedge S_{1} \cap S_{2}=k_{2}
$$

We observe that there exist an infinite sequence $\left(k_{n}, S_{n}\right): n \in \mathbb{N}$, such that $\left(k_{n}, S_{n}\right)<$ $\left(k_{n+1}, S_{n+1}\right)$. Indeed, choose an arbitrary face $S$ of $W$ and let $k$ be one of the edges of $S$. We set $k_{1}=k$ and $S_{1}=S$. Suppose that $\left(k_{n}, S_{n}\right)$ is constructed. Since $S_{n}$ has a center of symmetry, say $O_{n}$, then as $k_{n+1}$ we take the edge of $S_{n}$ symmetric to $k_{n}$ with respect to $O_{n}$. But each edge of $W$ is a common edge of exactly two faces, then there exist exactly one face $S_{n+1}$ of $W$ such that $S_{n} \cap S_{n+1}=k_{n+1}$. Clearly $\left(k_{n}, S_{n}\right)<\left(k_{n+1}, S_{n+1}\right)$. Since the set of all faces of $W$ is finite, the sequence $\left(k_{n}, S_{n}\right): n \in \mathbb{N}$ is periodic. Hence there exist (the smallest) $p \in \mathbb{N}$ such that $\left(k_{n+p}, S_{n+p}\right)=\left(k_{n}, S_{n}\right)$. Clearly, the edges of the sequence $k_{n}$ are parallel to each other, i.e. $k_{1}\left\|k_{2}, k_{2}\right\| k_{3}, \ldots k_{p-1} \| k_{p}$. The sum $T:=\bigcup_{i=1}^{n} S_{i}$ separates the boundary of $W$ onto two parts $W^{-}$and $W^{+}$. On the other hand the lengths of $k_{i}$ are equal, hence these edges define a translation $t$. The union $t\left(W^{-}\right) \cup W^{+}$defines a new polyhedron $W^{*}$ such that $W=W^{*}+I$ where $I$ is a diangle. This new polyhedron $W^{*}$ has still symmetric faces since each face $S^{*}$ of $W^{*}$ is a face of $W$. Moreover the number of edges of $W^{*}$ is smaller then the number of edges of $W$. This gives a possibility to use the induction argument. Thus we have proved, that if each face of $W$ is a centrally symmetric polygon, then $W$ is a sum of diangles. The converse is trivially true, because each face of $W$ is, up to an isometry, a Minkowski sum of a number of coplanar diangles.

Remark 4 If $W$ is a convex polyhedron in $\mathbb{R}^{3}$ and $A$ is a vertex of $W$, one may count the number of edges which meets at $A$. If $W$ has the dimension three, then at each vertex $A$ of $W$ meets at least three edges, but possibly more. However we have the following

Theorem 17 If $W$ is a Minkowski polyhedron, then at each vertex of $W$ meet at most three edges of $W$. 
Proof This may be proved using the induction argument with respect to the number of diangles generating the polyhedron. Suppose that our theorem is true for each positive polyhedron which is a sum of $k-1$ diangles. Take into account an ordinary polyhedron $W_{k}=\sum_{i=1}^{k} x_{i} I_{v_{i}}$ and choose a diangle $I_{v_{k}}$. Let $P_{k}$ denote the orthogonal projection parallel to $I_{v_{k}}$. The image $P_{k}\left(W_{k}\right)$ is a centrally symmetric polygon. Let $A_{1}, A_{2}, \ldots, A_{p}$ denote the vertices of $W_{k}$. The points $P\left(A_{i}\right)$ lies in the interior or in the boundary of $P_{k}\left(W_{k}\right)$. If $P_{k}\left(A_{i}\right)$ lies in the interior of $P_{k}\left(W_{k}\right)$ then $P_{k}\left(A_{i}\right)$ is also a projection of a vertex of $W_{k-1}$ and thus at $A_{i}$ meet only three edges (by induction hypothesis). If $P_{k}\left(A_{i}\right)$ lies on the boundary of $P_{k}\left(W_{k}\right)$ then at $P_{k}\left(A_{i}\right)$ meet two edges of $P_{k}\left(W_{k}\right)$ and in consequence three edges of $W_{k}$.

Open Access This article is distributed under the terms of the Creative Commons Attribution 4.0 International License (http://creativecommons.org/licenses/by/4.0/), which permits unrestricted use, distribution, and reproduction in any medium, provided you give appropriate credit to the original author(s) and the source, provide a link to the Creative Commons license, and indicate if changes were made.

\section{References}

1. Bochner, S.: Integration von Functionen, deren werte die Elemente eines Vectorraumes sind. Fundam. Math. 20, 262-267 (1933)

2. Ciesielska, D., Ciesielski, K.: On polyhedra with centrally symmetric faces. J. Nat. Geom. 10, 149-156 (1996)

3. Fenchel, W.: Generalization du theoreme de Brunn-Minkowski concernant les corps convexes. Comptes Rendus Acad. Sci. Paris 203, 764-766 (1936)

4. Hörmander, L.: Sur la fonction d'appui des ensembles convexes dans un espace localement convexe. Ark. Mat. 3, 181-186 (1954)

5. Kakutani, S.: Concrete representation of abstract (M)-spaces. Ann. Math. (2) 42, 994-1024 (1941)

6. Radström, H.: An embedding theorem for spaces of convex sets. Proc. Am. Math. Soc. 3(1), 165-169 (1952)

7. Szafraniec, F.H.: The Reproducing Kernel Property and Its Space: The Basics. Springer, Berlin (2015)

8. Tutaj, E.: An example of a reproducing kernel Hilbert space. Complex Anal. Oper. Theory (2018). https://doi.org/10.1007/s11785-018-0761-1

9. Tutaj, E.: Some particular norm in the Sobolev space $H^{1}[A, B]$. Complex Anal. Oper. Theory (2019). https://doi.org/10.1007/s11785-019-00893-3

Publisher's Note Springer Nature remains neutral with regard to jurisdictional claims in published maps and institutional affiliations. 